\title{
Association of Early and Late Contrast-Associated Acute Kidney Injury and Long-Term Mortality in Patients Undergoing Coronary Angiography
}

\author{
Zhubin Lun, ${ }^{1,2,3}$ Jin Liu, ${ }^{3}$ Liwei Liu, ${ }^{3}$ Jingjing Liang, ${ }^{4}$ Guanzhong Chen $\left(\mathbb{D},{ }^{3}\right.$ Shiqun Chen (i), ${ }^{3}$ \\ Bo Wang, ${ }^{3}$ Qiang Li, ${ }^{3}$ Haozhang Huang, ${ }^{3}$ Zhidong Huang, ${ }^{3}$ Danyuan $\mathrm{Xu},{ }^{3}$ Yunzhao Hu, \\ Ning Tan, ${ }^{3}$ Jiyan Chen, ${ }^{3}$ Yong Liu $(\mathbb{D})^{3}$ and Jianfeng Ye ${ }^{1}{ }^{1}$ \\ ${ }^{1}$ Department of Cardiology, Dongguan TCM Hospital, Dongguan 523000, China \\ ${ }^{2}$ The First School of Clinical Medicine, Guangdong Medical University, Zhanjiang 523808, China \\ ${ }^{3}$ Department of Cardiology, Guangdong Provincial Key Laboratory of Coronary Heart Disease Prevention, \\ Guangdong Cardiovascular Institute, Guangdong Provincial People's Hospital, Guangdong Academy of Medical Sciences, \\ Guangzhou 510080, China \\ ${ }^{4}$ Department of Cardiology, Shunde Hospital of Southern Medical University, Shunde, Guangzhou, China
}

Correspondence should be addressed to Yong Liu; liuyong@gdph.org.cn and Jianfeng Ye; yipjf@hotmail.com

Received 1 November 2020; Revised 26 January 2021; Accepted 24 February 2021; Published 8 March 2021

Academic Editor: Christian J. Mussap

Copyright (C) 2021 Zhubin Lun et al. This is an open access article distributed under the Creative Commons Attribution License, which permits unrestricted use, distribution, and reproduction in any medium, provided the original work is properly cited.

\begin{abstract}
Background. Contrast-associated acute kidney injury (CA-AKI) is a common complication in patients undergoing coronary angiography (CAG). However, few studies demonstrate the association between the prognosis and developed CA-AKI in the different periods after the operation. Methods. We retrospectively enrolled 3206 patients with preoperative serum creatinine (Scr) and at least twice SCr measurement after CAG. CA-AKI was defined as an increase $\geq 50 \%$ or $\geq 0.3 \mathrm{mg} / \mathrm{dL}$ from baseline in the 72 hours after the procedure. Early CA-AKI was defined as having the first increase in SCr within the early phase ( $<24$ hours), and late CA-AKI was defined as an increase in SCr that occurred for the first time in the late phase (24-72 hours). The first endpoint of this study was long-term all-cause mortality. Kaplan-Meier analysis was used to count the cumulative mortality, and the log-rank test was used to assess differences between curves. Univariate and multivariate cox regression analyses were performed to assess whether patients who developed different type CA-AKI were at increased risk of long-term mortality. Results. The number of deaths in the 3 groups was 407 for normal (12.7\%), 106 for early CA-AKI (32.7\%) and 57 for late CA-AKI (17.7\%), during a median follow-up period of 3.95 years. After adjusting for important clinical variables, early CA-AKI (HR $=1.33,95 \%$ CI: $1.02-1.74$, $P=0.038)$ was significantly associated with mortality, while late CA-AKI (HR $=0.92,95 \%$ CI: $0.65-1.31, P=0.633)$ was not. The same results were found in patients with coronary artery disease, chronic kidney disease, diabetes mellitus, and percutaneous coronary intervention. Conclusions. Early increases in Scr, i.e., early CA-AKI, have better predictive value for long-term mortality. Therefore, in clinical practice, physicians should pay more attention to patients with early renal injury related to long-term prognosis and give active treatment.
\end{abstract}

\section{Introduction}

Contrast-associated acute kidney injury (CA-AKI) is a common complication in patients undergoing coronary angiography (CAG), and CA-AKI is associated with prolonged hospitalization, long-term morbidity, and mortality [1-5].
The majority of studies on the long-term outcome of patients after angiography reported that CA-AKI was an independent predictor of mortality $[6,7]$. Notably, the predictive power of CA-AKI on outcome is related to different definitions, which commonly refer to the increase in the serum creatinine (SCr) level from baseline to 48-72 hours after contrast exposure [8]. In previous research, CA- 
AKI (defined as an increase of Scr $\geq 0.3 \mathrm{mg} / \mathrm{dL}$ or $\geq 50 \%$ from baseline) had higher population-attributable risks (PAR) for long-term mortality than other definitions of CA-AKI [9].

Recent evidence suggests that creatinine levels at different postoperative timepoints are related to adverse clinical outcomes [10-12]. Yong et al. observed that increased creatinine levels at 48-72 hours postsurgery have similar good predictive value for long-term mortality as early increases in creatine [13]. Ribichini et al. found that the increase of SCr in the early phase (12 hours) had better diagnostic power than other phases ( 24 and 48 hours) for predicting 30-day renal damage after exposure to contrast media $[14,15]$. However, in these studies, the association of the changes in postoperative creatinine levels in patients who developed CA-AKI with long-term mortality was limited, which reduced the ability to assess the predictive value of CA-AKI compared with different definitions. In addition, the definitions that are strongest predictors of mortality and their relationship with long-term outcome in the different time courses remain uncertain. Meanwhile, evidence for the influence of CA-AKI on prognosis is derived mainly from the ST-elevated myocardial infarction and acute myocardial infarction populations.

Therefore, it is desirable to evaluate the association between the occurrence of CA-AKI in different time periods and long-term mortality among patients undergoing CAG.

\section{Methods}

2.1. Enrollment and Treatment. This observational study included all consecutive patients who underwent CAG at Guangdong Provincial People's Hospital between January 2008 and December $2018(n=81,850)$. The patients aged $\geq 18$ years who agreed to remain at hospital to undergo monitoring for 3 days after CAG were included in the study. Exclusion criteria included missing preoperative SCr values, missing first $24 \mathrm{~h}$ SCr values, and 24-72 h SCr values after CAG. Patients who lack follow-up data were also excluded. There were in total 81,850 potentially eligible patients, of whom 3,206 had preoperative and postoperative Scr values and therefore could be included for analysis (Figure 1). The study was approved by the Ethics Committee of Guangdong Provincial People's Hospital.

In accordance with standard clinical practice, CAG was performed through the femoral or radial approach using standard guide catheters, guidewires, balloon catheters, and stents. The treatment of patients was based on guidelines from the American Heart Association/American College of Cardiology Foundation [16].

2.2. Endpoint and Definitions. The endpoint of this study was long-term all-cause mortality. All eligible patients included were retrospectively followed up through office visits or telephone interviews. CA-AKI defined as an increase $\geq 50 \%$ or $\geq 0.3 \mathrm{mg} / \mathrm{dL}$ from baseline in the 72 hours after the procedure [17]. Early CA-AKI was defined as having the first increase in SCr within the early phase ( $<24$ hours), and late CA-AKI was defined as an increase in SCr that occurred for the first time in the late phase (24-72 hours). The normal kidney function was defined as normal kidney function or patients with increase $<50 \%$ or $<0.3 \mathrm{mg} / \mathrm{dL}$ in creatinine in the 72 hours after angiography. The estimated glomerular filtration rate (eGFR) was calculated by applying the Modification of Diet in Renal Disease (MDRD) equation. Chronic kidney disease (CKD) was defined as an $\mathrm{eGFR}<60 \mathrm{~mL} / \mathrm{min} / 1.73 \mathrm{~m}^{2}$. Anemia was defined as a baseline hematocrit value $<39 \%$ for men or $<36 \%$ for women according to the World Health Organization criteria. Congestive heart failure (CHF) was defined as New York Heart Association (NYHA) functional class $>2$, Killip class $>1$, or pulmonary edema. Diabetes mellitus (DM) was defined as a previous diagnosis of diabetes or an $\mathrm{HbAlc}$ level $\geq 6.5(48 \mathrm{mmol} / \mathrm{mol})$.

2.3. Statistical Analysis. According to the endpoints, all patients were divided into three groups (normal, early CA$\mathrm{AKI}$, and late CA-AKI). Continuous variables were compared using one-way analysis of variance (ANOVA) and presented as the mean \pm SD or median \pm IQR. The Pearson chi-squared test was used to analyze categorical data, and the categorical data are expressed as counts (percentages). Kaplan-Meier (K-M) analysis was used to determine the cumulative mortality, and a log-rank test was used to assess differences between curves. Univariate and multivariate Cox regression analyses were performed to assess whether patients who developed different types of CA-AKI had an increased risk of long-term death. The data were analyzed based on available cases, and missing data were not included. For multivariate models, cases with missing values for included factors were excluded listwise. Candidate predictors that were significantly different at $P<0.05$ in univariate analysis for CA-AKI versus no CA-AKI and were clinically relevant were included in the Cox regression models. Subgroup analyses were conducted in patients with coronary artery disease (CAD), CKD, and DM. Sensitivity analysis was performed using an additional definition of CA-AKI (defined as an increase $\geq 25 \%$ or $\geq 0.5 \mathrm{mg} / \mathrm{dL}$ from baseline in the 72 hours after the procedure). All data analyses were performed using $R$ software (version 3.6.5; $R$ Foundation for Statistical Computing, Vienna, Austria). A 2-tailed $P$ value $<0.05$ indicated significance for all analyses.

\section{Results}

3.1. Clinical and Procedural Characteristics. A total of 3,206 consecutive patients who underwent CAG were included in the study (Figure 1). The patients were divided into three different groups: 2,530 (78.91\%) patients with no CA-AKI, 354 (11.04\%) patients with early CA-AKI and $322(10.04 \%)$ patients with late CA-AKI. Additionally, there were $668(20.85 \%)$ patients with acute myocardial infarction (AMI), 498 (15.53\%) patients with chronic kidney disease (CKD), $886(27.61 \%)$ patients with diabetes mellitus (DM), and 849 (26.69\%) patients with chronic heart failure (CHF). The usage rates of ACEIs/ARBs, betablockers, statins, and diuretics were 53.05\%, 60.67\%, 


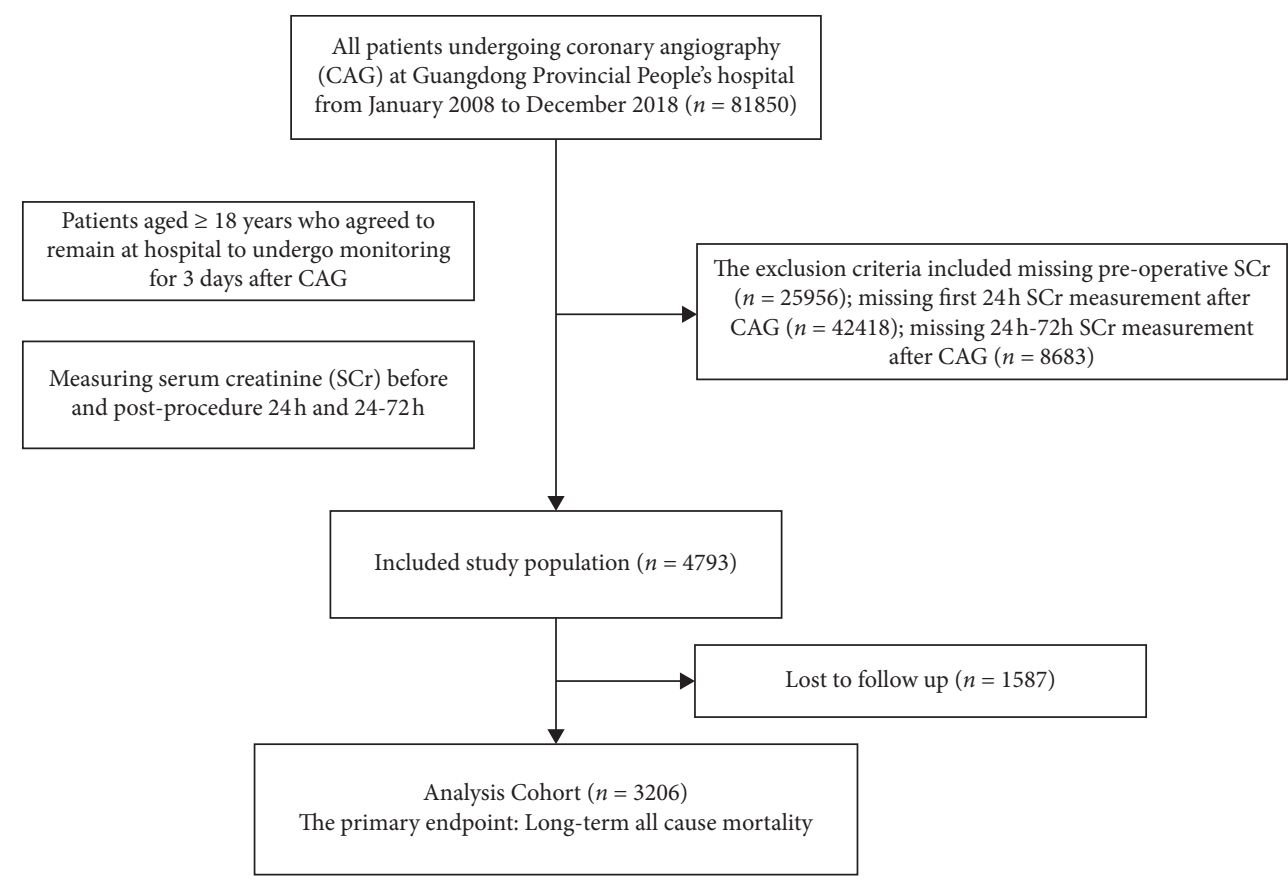

Figure 1: Study flow chart.

$60.09 \%$, and $42.89 \%$, respectively. Overall, the mean age was $63.75 \pm 11.02$ years, and men made up $70.84 \%$ of the total population (Table 1).

3.2. Primary Outcomes. During the follow-up period (median, 3.95 years), 570 patient deaths occurred. The number of deaths in the 3 groups was 407 for normal (12.7\%), 106 for early CA-AKI (32.7\%), and 57 for late CA-AKI (17.7\%). Kaplan-Meier curve analysis revealed that there are significant statistical differences between normal, early CAAKI, and late CA-AKI $(P<0.001$, Figure 2$)$. In the Cox proportional hazards model, after adjustment for age $\geq 75$, male, acute myocardial infarction, chronic heart failure, hypertension, anemia, contrast media volume, beta-blocker uses, statin use, and diuretic uses, early CA-AKI was significantly associated with mortality (HR: 1.33, 95\% CI: 1.02-1.74, $P=0.038$ ), while late CA-AKI was not (HR: 0.92, 95\% CI:0.65-1.31, $P=0.633$ ) (Table 2 and Figure 3 ).

Our results showed that early CA-AKI and late CA-AKI were significantly associated with in-hospital death after adjusting age, chronic heart failure, and acute myocardial infarction (OR: 9.95, 95\% CI: 4.70-21.04, $P<0.001$ and OR: 4.75, 95\% CI: $1.84-12.28, P=0.001$, respectively) (Supplement Table 1).

3.3. Sensitivity Analysis. We conducted the same analysis in patients with CAD and found that early CA-AKI was significantly correlated with long-term mortality (HR: 1.39, 95\% CI: $1.04-1.87, P=0.028)$, while late CA-AKI was not significantly correlated (HR: 1.21, 95\% CI: 0.84-1.76, $P=0.307)$. At the same time, we found similar results in patients with DM, CKD, or PCI. However, we found that early and late CA-AKI were not associated with long-term mortality in non-PCI patients (HR: $1.30,95 \%$ CI: 0.89-1.92, $P=0.179$ and HR: $4.75,95 \% \mathrm{CI}: 1.84-12.28, P=0.001$, respectively) (Figure 4).

When using different criteria, e.g., CA-AKI $\mathrm{I}_{0525}$ (an increase $\geq 25 \%$ or $\geq 0.5 \mathrm{mg} / \mathrm{dL}$ from baseline in the 72 hours after the procedure), Kaplan-Meier curve analysis revealed that there are significant statistical differences between normal, early CA-AKI, and late CA-AKI $(P<0.001$, Figure 5).

\section{Discussion}

We compared the relationship between the onset of CA-AKI and long-term mortality in patients undergoing CAG. According to our analysis, patients with early CA-AKI had a worse prognosis than patients without CA-AKI. Moreover, we also found similar results in CA-AKI which defined as a serum creatine elevation $\geq 25 \%$ or $\geq 0.5 \mathrm{mg} / \mathrm{dl}$ within 72 hours. Precise interventions for high-risk groups are of great significance for reducing mortality, medical resource use, and medical costs in clinical practice.

In our cohort, the incidence of CA-AKI was 21.09\%, which was higher than the incidence reported by George Chalikias [18]. At a median follow-up of 3.95 years, the mortality was $17.78 \%$, which was slightly lower than the mortality reported by Sinkovic et al. [19]. Sinkovic included patients with STEMI in the study, which may have led to increased mortality. When using the definition of CA-AKI as a serum creatine elevation $\geq 50 \%$ or $\geq 0.3 \mathrm{mg} / \mathrm{dl}$ within 72 hours, we unexpectedly found that early CA-AKI can increase the risk of long-term mortality, while the long-term prognosis of late CA-AKI was similar to that of patients without CA-AKI. This means that patients with CA-AKI within 24 hours after surgery have a poor long-term 
TABLE 1: Baseline characteristics.

\begin{tabular}{|c|c|c|c|c|c|}
\hline Characteristic & Overall $(n=3,206)$ & Normal $(n=2,530)$ & Late CA-AK $(n=322)$ & Early CA-AKI $(n=354)$ & $P$ value \\
\hline Age, years & $63.75(11.02)$ & $63.61(10.97)$ & $63.52(10.54)$ & $64.89(11.73)$ & 0.1148 \\
\hline Age $>75$, years $(\%)$ & $584(18.22)$ & $441(17.43)$ & $57(17.70)$ & $86(24.29)$ & 0.0071 \\
\hline Men, $n(\%)$ & $2271(70.84)$ & $1828(72.25)$ & $191(59.32)$ & $252(71.19)$ & $<0.001$ \\
\hline Diabetes mellitus, $n(\%)$ & $886(27.64)$ & $717(28.34)$ & $76(23.60)$ & $93(26.27)$ & 0.1673 \\
\hline AMI, $n(\%)$ & $668(20.85)$ & $542(21.44)$ & $45(13.98)$ & $81(22.88)$ & 0.023 \\
\hline Hypertension, $n(\%)$ & $1744(54.40)$ & $1408(55.65)$ & $132(40.99)$ & $204(57.63)$ & $<0.001$ \\
\hline $\mathrm{CKD}, n(\%)$ & $498(15.53)$ & $342(16.85)$ & $67(8.15)$ & $89(25.14)$ & $<0.001$ \\
\hline $\mathrm{CHF}, n(\%)$ & $849(26.69)$ & $616(24.49)$ & $111(34.91)$ & $122(35.06)$ & $<0.0001$ \\
\hline $\mathrm{CAD}, n(\%)$ & $2371(73.98)$ & $1968(77.82)$ & $163(50.62)$ & $240(67.80)$ & $<0.001$ \\
\hline PCI, $n(\%)$ & $1599(49.88)$ & $1365(53.95)$ & $97(30.12)$ & $137(38.70)$ & $<0.001$ \\
\hline Vascular disease, $n(\%)$ & $713(22.25)$ & $595(23.54)$ & $54(16.77)$ & $64(18.08)$ & $<0.001$ \\
\hline Anemia, $n(\%)$ & $161(5.06)$ & $115(4.61)$ & $19(5.94)$ & $31(8.81)$ & $<0.001$ \\
\hline $\mathrm{CMV}, \mathrm{ml}$ & $163.94(112.43)$ & $169.90(112.32)$ & $119.10(98.03)$ & $162.15(116.40)$ & $<0.001$ \\
\hline LDLC, $\mathrm{mmol} / \mathrm{L}$ & $2.79(0.96)$ & $2.81(0.99)$ & $2.72(0.85)$ & $2.78(0.88)$ & 0.3550 \\
\hline $\mathrm{HDLC}, \mathrm{mmol} / \mathrm{L}$ & $0.99(0.28)$ & $0.99(0.28)$ & $1.03(0.31)$ & $0.99(0.28)$ & 0.0485 \\
\hline Cys-C, mg/L & $1.43(0.81)$ & $1.36(0.74)$ & $1.69(0.91)$ & $1.95(1.18)$ & $<0.0001$ \\
\hline $\mathrm{eGFR}, \mathrm{ml} / \mathrm{min} / 1.73 \mathrm{~m}^{2}$ & $68.74(28.58)$ & $69.59(27.83)$ & $66.82(29.83)$ & $64.38(32.07)$ & 0.0025 \\
\hline Creatine kinase, U/L & $261.66(598.67)$ & $92.00(59.05,164.50)$ & $82.00(54.00,191.25)$ & $96.00(59.00,176.30)$ & 0.2441 \\
\hline \multirow{2}{*}{ NT-proBNP, pg/mL } & \multirow{2}{*}{3724.79 (7141.08) } & $877.50(187.15,3045.00)$ & $1359.00(527.80,4260.00)$ & $1684.00(536.80,5173.00)$ & \multirow[t]{2}{*}{$<0.001$} \\
\hline & & $877.50(187.15,3045.00)$ & $1359.00(527.80,4260.00)$ & $1684.00(536.80,5173.00)$ & \\
\hline NYHA class $>1$ & $1791(92.22)$ & $1373(91.29)$ & $216(94.74)$ & $202(96.19)$ & 0.0147 \\
\hline Beta-blocker use & $1839(60.67)$ & $1515(62.17)$ & $149(51.20)$ & $175(57.76)$ & $<0.001$ \\
\hline Statin use & $2094(69.09)$ & $1817(74.56)$ & $108(37.11)$ & $169(55.78)$ & $<0.001$ \\
\hline Diuretic use & $1300(42.89)$ & $893(36.64)$ & $219(75.26)$ & $188(62.05)$ & $<0.001$ \\
\hline ACEI/ARB use & $1608(53.05)$ & $1400(57.45)$ & $93(31.96)$ & $115(37.95)$ & $<0.001$ \\
\hline
\end{tabular}

Abbreviations: AMI, acute myocardial infarction; CKD, chronic kidney disease; CHF, chronic heart failure; CAD, coronary artery disease; HDL-C, highdensity lipoprotein cholesterol; LDL-C, low-density lipoprotein cholesterol; LVEF, left ventricular ejection fraction; eGFR, estimated glomerular filtration rate; BUN, blood urea nitrogen; PCI, percutaneous coronary intervention; CMV, contrast media volume; ACEI/ARB, angiotensin-converting enzyme inhibitor/angiotensin receptor blocker.

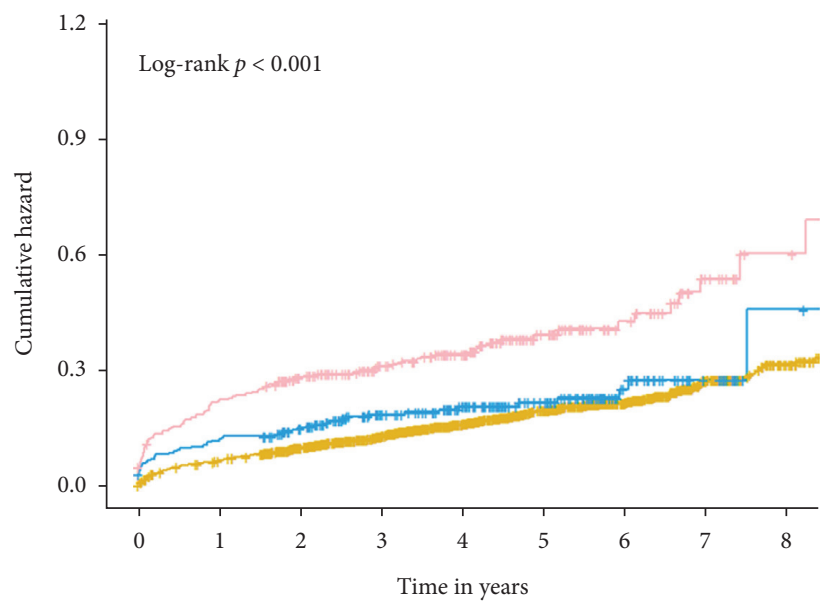

Number at risk

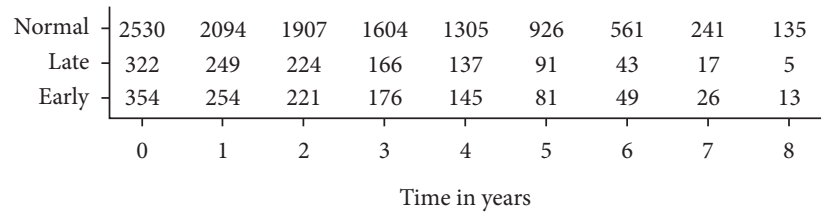

$$
\begin{array}{ll}
+1 & \text { Normal } \\
+ \text { Late } \\
+ \text { Early }
\end{array}
$$

FIGURE 2: Kaplan-Meier curves for the cumulative probability of mortality stratified according to early CA-AKI, late CA-AKI, and normal. CA-AKI is defined as an increase $\geq 50 \%$ or $\geq 0.3 \mathrm{mg} / \mathrm{dL}$ from baseline. 
TABLE 2: Univariable and multivariable analysis of risk factors for long-term mortality.

\begin{tabular}{|c|c|c|c|c|c|c|}
\hline & \multicolumn{3}{|c|}{ Univariable analysis } & \multicolumn{3}{|c|}{ Multivariable analysis } \\
\hline & HR & $95 \% \mathrm{CI}$ & $P$ value & HR & $95 \% \mathrm{CI}$ & $P$ value \\
\hline Age $\geq 75$ & 2.52 & $2.12-3.00$ & $<0.001$ & 2.28 & $1.86-2.80$ & $<0.001$ \\
\hline Male & 1.28 & $1.06-1.55$ & 0.011 & 1.39 & $1.12-1.73$ & 0.003 \\
\hline $\mathrm{CHF}$ & 2.46 & $2.08-2.91$ & $<0.001$ & 1.84 & $1.49-2.28$ & $<0.001$ \\
\hline AMI & 1.55 & $1.29-1.87$ & $<0.001$ & 0.90 & $0.71-1.13$ & 0.359 \\
\hline Anemia & 2.64 & $2.03-3.45$ & $<0.001$ & 1.91 & $1.40-2.60$ & $<0.001$ \\
\hline Hypertension & 1.57 & $1.32-1.87$ & $<0.001$ & 1.52 & $1.23-1.88$ & $<0.001$ \\
\hline Beta-blocker & 1.04 & $0.86-1.25$ & 0.697 & 1.01 & $0.83-1.23$ & 0.900 \\
\hline Diuretic & 1.76 & $1.47-2.12$ & $<0.001$ & 1.75 & $1.42-2.15$ & $<0.001$ \\
\hline Statin & 1.52 & $1.21-1.92$ & $<0.001$ & 1.37 & $1.05-1.79$ & 0.020 \\
\hline CMV & 1.00 & $1.00-1.00$ & 0.514 & 1.00 & $1.00-1.00$ & 0.769 \\
\hline \multicolumn{7}{|l|}{ CA-AKI } \\
\hline Normal & Ref & Ref & Ref & Ref & Ref & Ref \\
\hline Late & 2.21 & $0.97-1.69$ & 0.078 & 0.92 & $0.65-1.31$ & 0.633 \\
\hline Early & 1.28 & $1.78-2.73$ & $<0.001$ & 1.33 & $1.02-1.31$ & 0.038 \\
\hline
\end{tabular}

Abbreviations: AMI, acute myocardial infarction; CHF, chronic heart failure; CMV, contrast media volume; CA-AKI, contrast-associated acute kidney injury.

\begin{tabular}{|c|c|c|c|c|c|c|c|c|}
\hline Variable & Patients & $\mathrm{HR}(95 \% \mathrm{CI})$ & P-value & \multicolumn{3}{|c|}{ Hazard ratio } & & \\
\hline CA-AKI(Unadjusted) & $570 / 3206$ & & & & & & & \\
\hline Late vs. normal & & $1.28(0.97,1.69)$ & 0.078 & & & & & \\
\hline Early vs. normal & & $2.21(1.78,2.73)$ & $<0.001^{*}$ & & & & & \\
\hline CA-AKI(Adjusted) & $453 / 2986$ & & & & & & & \\
\hline Late vs. normal & & $0.92(0.65,1.31)$ & 0.633 & & & & & \\
\hline \multirow[t]{2}{*}{ Early vs. normal } & & $1.33(1.02,1.74)$ & $0.038^{*}$ & & & & & \\
\hline & & & & 0.5 & 1 & 1.5 & 2 & 2.5 \\
\hline
\end{tabular}

Figure 3: Multivariable analysis for mortality stratified according to early, late, and normal. Adjusted for age $\geq 75$, male, acute myocardial infarction, chronic heart failure, hypertension, anemia, contrast media volume, beta-blocker uses, statin use, and diuretic uses; CA-AKI defined as an increase $\geq 50 \%$ or $\geq 0.3 \mathrm{mg} / \mathrm{dL}$ from baseline.

prognosis. In previous studies, early CA-AKI was shown to increase the risk for long-term mortality, and the results showed that late CA-AKI was significantly related to longterm mortality, which contradicts our conclusions [13]. The possible reason is that the median follow-up time of Liu et al.'s study was 2.45 years, while the median follow-up time of our study was 3.95 years. After prolonging the follow-up time, patients with late CA-AKI no longer had an increased mortality rate.

After exposure to contrast media, kidney damage can occur due to early hemodynamic instability, and 4-6 hours after the procedure is when contrast media excretion occurs $[5,20,21]$. Contrast media damages renal tubular epithelial cells, leading to loss of function, apoptosis, and necrosis [5]. $\mathrm{SCr}$ is a clinically significant and sensitive indicator of renal function, and an increase in SCr is associated with poor long-term and short-term outcomes [22]. Ribichini et al. found that the early increase of SCr (12 hours from baseline) had better diagnostic power for predicting short-term kidney damage, but they did not discuss the relationship with long-term prognosis [15]. At present, the new diagnosis and treatment technology allows for patients to be discharged within 24 hours after CAG, which may affect the assessment of the poor prognosis of CA-AKI. Therefore, patients with early CA-AKI should receive additional renal treatment. The long-term prognosis of late CA-AKI patients is similar to that of patients without CA-AKI. This indicates that CA-AKI on the second and third days after surgery will not affect the prognosis and should not interfere with the current treatment plan.

In the subgroup analysis, when we adjusted for some important clinical variables (such as $\mathrm{CKD}, \mathrm{DM}$, and $\mathrm{CAD}$ ), we also found that only early CA-AKI was associated with long-term prognosis. When we adjusted for multiple baseline variables, the results remain unchanged, which indicates that our results are consistent. Late CA-AKI is more likely to be related to creatinine fluctuations. When using the definition of CA-AKI as a serum creatine elevation $\geq 25 \%$ or $\geq 0.5 \mathrm{mg} / \mathrm{dl}$ within 72 hours, we found similar results, which further confirmed our conclusion. The clinical and research significance of this study is that we should focus on the SCr level on the first day after CAG surgery to identify the high-risk population.

Our research had several limitations. First, our study was a single-center retrospective study, which may have caused our results to be unrepresentative. However, the follow-up period of our study was 6 years, which made our research results more credible. Second, our outcome event was only long-term 


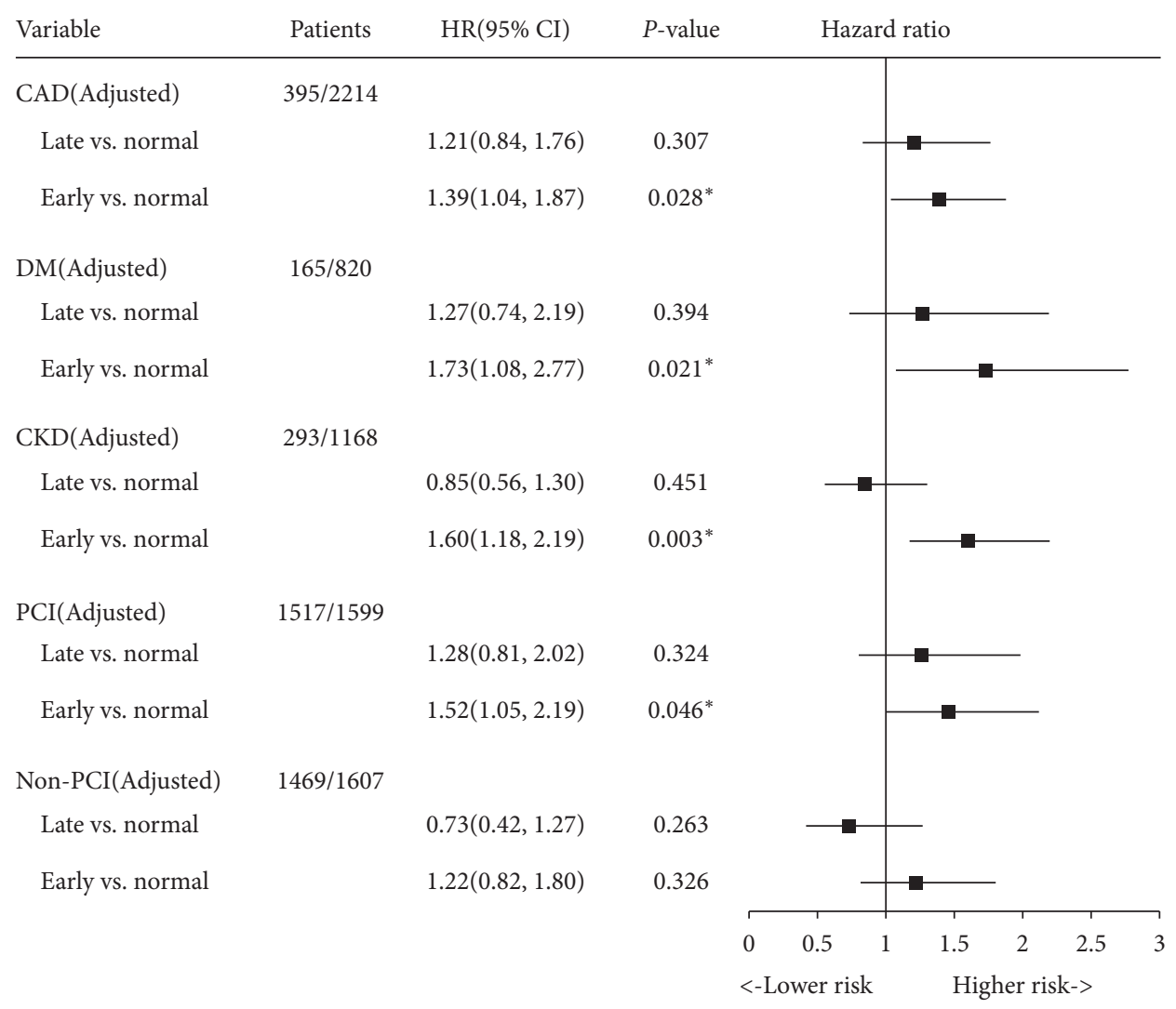

FIGURE 4: Multivariable analysis for mortality stratified according to early CA-AKI, late CA-AKI, and normal in patients with CAD, CKD, DM, PCI, and Non-PCI. CA-AKI defined as an increase $\geq 50 \%$ or $\geq 0.3 \mathrm{mg} / \mathrm{dL}$ from baseline. Adjusted for age $\geq 75$, male, acute myocardial infarction, chronic heart failure, hypertension, anemia, contrast media volume, beta-blocker uses, statin use, and diuretic uses.
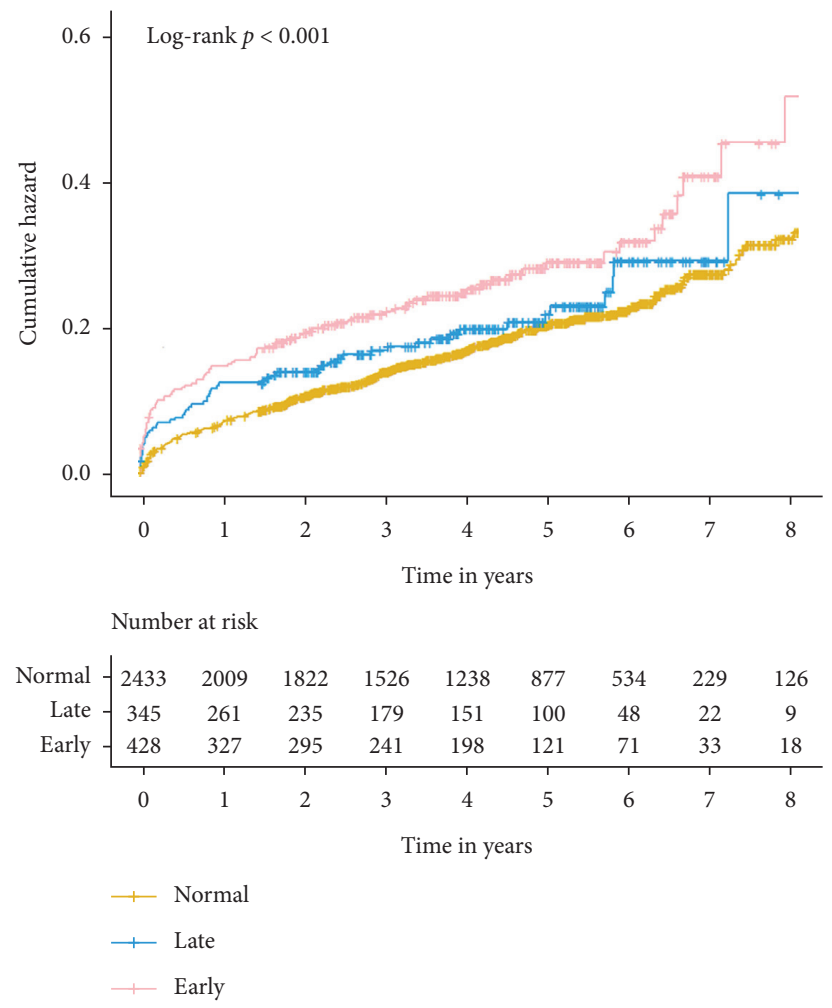

FIGURE 5: Kaplan-Meier curves for the cumulative probability of mortality stratified according to early CA-AKI, late CA-AKI, and normal. CA-AKI is defined as an increase $\geq 25$ or $\geq 0.5 \mathrm{mg} / \mathrm{dL}$ from baseline. 
follow-up mortality, and there was a lack of endpoints including in-hospital death, rehospitalization, and assessment of renal function during follow-up. These outcomes are unfavorable for short-term assessment of patients' health-related economic burden and long-term assessment of the impact on all-cause mortality. Third, we defined early CA-AKI and late CA-AKI based on previous research, which may have affected our conclusions. However, after adjusting for important clinical variables, we found similar results in other populations (CAD, CKD, and DM), which indicates that our results are credible. Fourth, adequate periprocedural IV saline hydration for the prevention of CA-AKI should was recommended in previous guidelines [23]. Since we are unable to obtain the patient's hydration data from the hospital system, we cannot adjust the hydration in the multivariable analysis. But, we have adjusted some important risk factors at the same time, which will make our results more credible.

\section{Conclusion}

Our results confirm that early CA-AKI patients are significantly associated with long-term mortality after CAG, while late CA-AKI patients are not significantly associated with long-term mortality. This means that in clinical practice, physicians should pay more attention to patients with early CA-AKI and provide active treatment.

\section{Data Availability}

The datasets used and/or analyzed during this study are available from the corresponding author on reasonable request.

\section{Disclosure}

The funders had no role in the study design, data collection and analysis, decision to publish, or preparation of the manuscript; the work was not funded by any industry sponsors.

\section{Conflicts of Interest}

The authors declare that there are no conflicts of interest.

\section{Authors' Contributions}

NT, JYC, YL, YX, and JFY were involved in conception and design of research. ZBL, JL, LWL, JJL, GZC, BW, QL, HZH, and $\mathrm{ZDH}$ were responsible for data acquisition. $\mathrm{ZBL}, \mathrm{JL}$, SQC, and DYX were involved in data analysis and evaluation. ZBL, JL, and LWL drafted the manuscript. YZH, PYC, YL, and JFY were responsible for manuscript critical revision for key intellectual content. Zhubin Lun, Jin Liu, and Liwei Liu contributed equally to this work.

\section{Acknowledgments}

Special thanks to Professor Ying Xian (Duke Clinical Research Institute, Durham, NC, 27705, USA) for helping with the study design. We thank Dr. Peng-yuan Chen for his dedication and support. In addition, we want to thank, in particular, the patience, care, and love from Xiaoyu Huang for a long time. This study was supported by grants from the Beijing Lisheng Cardiovascular Health Foundation and Guangdong Provincial People's Hospital Foundation (no. LHJJ20141751), Progress in Science and Technology Project of Guangzhou (grant no. 201904010470), the Science and Technology Planning Project of Guangzhou (grant no. 201704020124), and Dengfeng Project in Guangdong Province (DFJH201919 and DFJH2020026).

\section{Supplementary Materials}

Supplement Table 1: univariable and multivariable analyses of risk factors for in-hospital death. (Supplementary Materials)

\section{References}

[1] C. S. Rihal, S. C. Textor, D. E. Grill et al., "Incidence and prognostic importance of acute renal failure after percutaneous coronary intervention," Circulation, vol. 105, no. 19, pp. 2259-2264, 2002.

[2] S. D. Weisbord, H. Chen, R. A. Stone et al., "Associations of increases in serum creatinine with mortality and length of hospital stay after coronary angiography," Journal of the American Society of Nephrology, vol. 17, no. 10, pp. 2871-2877, 2006.

[3] A. M. From, B. J. Bartholmai, A. W. Williams, S. S. Cha, and F. S. McDonald, "Mortality associated with nephropathy after radiographic contrast exposure," Mayo Clinic Proceedings, vol. 83, no. 10, pp. 1095-1100, 2008.

[4] A. M. Mitchell, J. A. Kline, A. E. Jones, and J. A. Tumlin, "Major adverse events one year after acute kidney injury after contrast-enhanced computed tomography," Annals of Emergency Medicine, vol. 66, no. 3, pp. 267-274, 2015.

[5] R. Mehran, G. D. Dangas, and S. D. Weisbord, "Contrastassociated acute kidney injury," New England Journal of Medicine, vol. 380, no. 22, pp. 2146-2155, 2019.

[6] P. Roy, V. Raya, T. Okabe et al., "Incidence, predictors, and outcomes of post-percutaneous coronary intervention nephropathy in patients with diabetes mellitus and normal baseline serum creatinine levels," The American Journal of Cardiology, vol. 101, no. 11, pp. 1544-1549, 2008.

[7] J. Wi, Y.-G. Ko, J.-S. Kim et al., "Impact of contrast-induced acute kidney injury with transient or persistent renal dysfunction on long-term outcomes of patients with acute myocardial infarction undergoing percutaneous coronary intervention," Heart, vol. 97, no. 21, pp. 1753-1757, 2011.

[8] B. Guillon, F. Ecarnot, C. Marcucci et al., "Incidence, predictors, and impact on six-month mortality of three different definitions of contrast-induced acute kidney injury after coronary angiography," The American Journal of Cardiology, vol. 121, no. 7, pp. 818-824, 2018.

[9] L. Lei, Y. Xue, Z. Guo et al., "A comparison between different definitions of contrast-induced acute kidney injury for longterm mortality in patients with acute myocardial infarction," IJC Heart and Vasculature, vol. 28, p. 100522, 2020.

[10] J. Kooiman, P. A. Le Haen, G. Gezgin et al., "Contrast-induced acute kidney injury and clinical outcomes after intra-arterial and intravenous contrast administration: risk comparison adjusted for patient characteristics by design," American Heart Journal, vol. 165, no. 5, pp. 793-799, 2013. 
[11] A. Narula, R. Mehran, G. Weisz et al., "Contrast-induced acute kidney injury after primary percutaneous coronary intervention: results from the HORIZONS-AMI substudy," European Heart Journal, vol. 35, no. 23, pp. 1533-1540, 2014.

[12] Y. Han, G. Zhu, L. Han et al., "Short-term rosuvastatin therapy for prevention of contrast-induced acute kidney injury in patients with diabetes and chronic kidney disease," Journal of the American College of Cardiology, vol. 63, no. 1, pp. 62-70, 2014.

[13] Y. Liu, C. Y. Duan, K. Wang et al., "Could late measurement of serum creatinine be missed for patients without early increase in serum creatinine following coronary angiography?," Medicine, vol. 96, no. 50, Article ID e8460, 2017.

[14] F. Ribichini, M. Graziani, G. Gambaro et al., "Early creatinine shifts predict contrast-induced nephropathy and persistent renal damage after angiography," The American Journal of Medicine, vol. 123, no. 8, pp. 755-763, 2010.

[15] F. Ribichini, G. Gambaro, M. S. Graziani et al., "Comparison of serum creatinine and cystatin $\mathrm{C}$ for early diagnosis of contrast-induced nephropathy after coronary angiography and interventions," Clinical Chemistry, vol. 58, no. 2, pp. 458-464, 2012.

[16] R. S. Wright, J. L. Anderson, C. D. Adams et al., "ACCF/AHA focused update of the guidelines for the management of patients with unstable Angina/non-ST-elevation myocardial infarction (updating the 2007 guideline): a report of the American College of Cardiology foundation/American heart association task force on practice guidelines developed in collaboration with the American College of emergency physicians, society for cardiovascular angiography and interventions, and society of thoracic surgeons," Journal of the American College of Cardiology, vol. 57, no. 19, pp. 1920-1959, 2011.

[17] R. L. Mehta, J. A. Kellum, S. V. Shah et al., "Acute Kidney Injury Network: report of an initiative to improve outcomes in acute kidney injury," Critical Care, vol. 11, no. 2, p. R31, 2007.

[18] G. Chalikias, I. Drosos, and D. N. Tziakas, "Contrast-Induced acute kidney injury: an update," Cardiovascular Drugs and Therapy, vol. 30, no. 2, pp. 215-228, 2016.

[19] A. Sinkovic, K. Masnik, and M. Mihevc, "Predictors of acute kidney injury (AKI) in high-risk ST-elevation myocardial infarction (STEMI) patients: a single-center retrospective observational study," Bosnian Journal of Basic Medical Sciences, vol. 19, no. 1, pp. 101-108, 2019.

[20] N. V. Guitterez, A. Diaz, G. C. Timmis et al., "Determinants of serum creatinine trajectory in acute contrast nephropathy," Journal of Interventional Cardiology, vol. 15, no. 5, pp. 349354, 2002.

[21] E. Seeliger, M. Sendeski, C. S. Rihal, and P. B. Persson, "Contrast-induced kidney injury: mechanisms, risk factors, and prevention," European Heart Journal, vol. 33, no. 16, pp. 2007-2015, 2012.

[22] C. Budano, M. Levis, M. D'Amico et al., "Impact of contrastinduced acute kidney injury definition on clinical outcomes," American Heart Journal, vol. 161, no. 5, pp. 963-971, 2011.

[23] F. J. Neumann, M. Sousa-Uva, A. Ahlsson et al., "ESC/EACTS Guidelines on myocardial revascularization," European Heart Journal, vol. 40, no. 2, pp. 87-165, 2018. 